\title{
«Papers on French Seventeenth Century Literature», vol. XXXV, n. 68, R. Zaiser ed.
}

\section{Chiara Rolla}

\section{(2) OpenEdition}

1 Journals

\section{Edizione digitale}

URL: http://journals.openedition.org/studifrancesi/7909

DOI: 10.4000/studifrancesi.7909

ISSN: 2421-5856

\section{Editore}

Rosenberg \& Sellier

\section{Edizione cartacea}

Data di pubblicazione: 1 juillet 2009

Paginazione: 390-391

ISSN: 0039-2944

\section{Notizia bibliografica digitale}

Chiara Rolla, ««Papers on French Seventeenth Century Literature», vol. XXXV, n. 68, R. Zaiser ed.», Studi Francesi [Online], 158 (LIII | II) | 2009, online dal 30 novembre 2015, consultato il 19 janvier 2021. URL: http://journals.openedition.org/studifrancesi/7909 ; DOI: https://doi.org/10.4000/studifrancesi.7909

Questo documento è stato generato automaticamente il 19 janvier 2021.

\section{(c)}

Studi Francesi è distribuita con Licenza Creative Commons Attribuzione - Non commerciale - Non opere derivate 4.0 Internazionale. 


\title{
«Papers on French Seventeenth Century Literature», vol. XXXV, n. 68, R. Zaiser ed.
}

\author{
Chiara Rolla
}

\section{NOTIZIA}

«Papers on French Seventeenth Century Literature», vol. XXXV, n. 68, R. ZAISER ed., Tübingen, G. Narr, 2008, pp. 457.

1 Il $68^{\circ}$ volume dei «Papers» raccoglie gli atti del Convegno "Pierre Corneille et l'Europe" tenutosi a Parigi nel settembre 2006, il cui scopo era mettere in evidenza le reciproche influenze che legano la letteratura europea del Grand Siècle con il drammaturgo francese (A. NIDERST, Préface, p. 9) proprio nell'anno in cui si celebrava il quarto centenario della sua nascita (M. FUMAROLI, La fête qui n'a pas eu lieu, pp. 11-13). Introduce il volume il contributo di A. NIDERST (Sur la circulation des livres et des spectacles en Europe, pp. 17-27) nel quale si danno risposte al quesito che sta a monte della problematica posta dal Convegno: come circolavano nel xvil secolo libri, idee, spettacoli e come quindi Corneille ha potuto conoscere autori stranieri e a sua volta farsi da loro conoscere?

2 La prima e la seconda sezione, «L'Europe vers Corneille (l'Italie)», si aprono con il contributo di A. DUPRAT (L'art et le précepte: Corneille et l'aristotélisme européen, pp. 33-43), incentrato sull'aristotelismo corneliano così come emerge dall'analisi dei suoi testi teorici. La libertà e l'emancipazione che Corneille manifesta nei confronti dei dettati della Poetica aristotelica trovano in Castelvetro e nella sua Poetica d'Aristotele volgarizzata e sposta un corrispondente d'Oltralpe ( $\mathrm{E}$. HÉNIN, Corneille et Castelvetro: une lecture polémique des portraits de la poétique, pp. 45 65). Ancora agli scritti teorici corneliani è dedicato lo studio di G. GIORGI (Les théoriciens italiens et les remarques de Corneille sur le roman et sur les différences entre le théâtre et le roman, pp. 73-83), che si concentra in 
particolare sulle riflessioni del drammaturgo inerenti il romanzo e sul rapporto che intercorre, a livello teorico, tra la nascente poetica narrativa e quella teatrale. R. ZAISER (Corneille héritier de Trissino: "Sophonisbe" et la naissance de la tragédie moderne, pp. 89-102) analizza la Sophonisbe di Corneille in rapporto a quella del Trissino, nella cui eredità si inseriscono anche le pièces di Montchrestien e di Mairet. E. Garofalo (Corneille et la "Jérusalem délivrée", pp. 103-113) analizza i rapporti che legano Corneille al Tasso, mettendo in evidenza determinati espliciti riferimenti alla Gerusalemme liberata che si osservano in alcune pièces corneliane.

3 La terza sezione, «L'Europe vers Corneille (l'Espagne)», si apre con lo studio di C. MARCHAL-WEYL (Corneille et la 'comedia': la comédie cornélienne à l'épreuve du modèle espagnol, pp. 119-129): molte sono le affinità che legano il teatro di Corneille con quello spagnolo a lui contemporaneo, che si esprimono sia a livello teorico, nell'analoga indipendenza critica nei confronti di Aristotele, sia nell'esperienza pratica e nell'importanza accordata al piacere del pubblico. c. COUDERC (Corneille réécrit Lope de Vega: de "Amar sin saber a quién" à "La Suite du Menteur", pp. 131-142) studia il lavoro di adattamento che Corneille compie per redigere la Suite du Menteur partendo dalla comedia di Lope de Vega Amar sin saber a quién. Ancora al rapporto tra il drammaturgo francese e Lope de Vega, con particolare riguardo a quattro pièces - La Place Royale e La Suite du Menteur da un lato e La Boda entre dos maridos e Amar sin saber a quién dall'altro - è dedicato il contributo di M.F. SCHMIDT (Le thème de l'amitié, sa mise en situation dramatique et son évolution des "Noces aux deux maris" de Lope de Vega à "La Suite du Menteur" de Corneille, pp. 143-151), mentre lo studio di L. PICciola (Corneille et l'esprit de Gracián: une dramaturgie de la pointe, pp. 159-169) analizza l'influenza delle opere e dello spirito di Gracián sulla produzione corneliana.

4 La sezione «L'Europe vers Corneille (l'Angleterre)» comprende i contributi di F. LASSERRE (Contacts de Corneille avec le théâtre anglais, pp. 175-185) e di s. DOSMOND ("Clitandre", drame élisabéthain, pp. 191-198). Il primo rinviene in alcune opere del drammaturgo francese l'influsso del teatro inglese, non tanto shakespeariano, quanto quello di autori quali de Beaumont, Fletcher e Massinger; il secondo rileva invece in Clitandre una palese influenza del dramma elisabettiano in voga oltre Manica all'epoca in cui la tragicommedia francese in questione fu rappresentata.

5 Della sezione «En amont et en aval» fanno parte gli studi di A. SOARE ( $L a$ tragédie morale de l'action: de l'"Orazia" aux "Tegeaten" en passant par "Horace", pp. 209-223) e di D. BECKER ("Persée" et "Andromède" de Lope de Vega à Calderon de la Barca et de Corneille à Lully (1613-1682), pp. 225-233): Soare vede nell'Horace di Corneille la pièce francese più rappresentativa del genere «tragédie morale de l'action», che penetra in Europa grazie alle traduzioni in lingue romanze del xvi secolo dell'Elettra di Sofocle; Becker invece analizza le figure di Persée e di Andromède così come sono messe in scena in Spagna da Lope de Vega e da Calderon e in Francia da Corneille e, a fine secolo, da Lully e da Quinault.

6 "Corneille en Europe», titolo che raggruppa la sesta, settima ed ottava sezione, si apre con il contributo di E. BURY (Corneille et la République des Lettres européennes, pp. 237-249) dal quale emerge la posizione e il ruolo che il drammaturgo e le sue opere occupano all'interno del dibattito culturale contemporaneo. La ricezione di Corneille in Spagna, in Portogallo e in Polonia costituiscono rispettivamente l'oggetto degli articoli di c. DumAs (Corneille, le "Cid" et l'Espagne: des paradoxes après un 'coup de maître', pp. 251-261), 
di A.C. VIEGAS DOS SANTOS (La fortune de Corneille au Portugal ou les répercussions de la querelle $d u$ "Cid", pp. 267-277) e di I. MAMCZARZ (L'adaptation polonaise du "Cid" de Pierre Corneille par Jean André Morsztyn et sa représentation à la cour royale (1662), pp. 279-288). Y. GIRAUD (Un admirateur de Corneille dans l'Allemagne baroque: Andreas Gryphius et son "Horribilicribrifax", pp. 295-309) rinviene in un autore tedesco grande ammiratore di Corneille e suo contemporaneo - Andreas Gryphius - un'evidente influenza del francese sulla sua produzione teatrale ed in particolare sulla pièce Horribilicribrifax. Della traduzione italiana del teatro di Corneille che Baretti cura nel xviII secolo si occupa lo studio di N. CLERICI BALMAS (Giuseppe Baretti traducteur de Corneille, pp. 311-321), mentre M. DOGLIO MAZzoccHI mette diacronicamente a confronto Corneille con Alfieri (Corneille et Alfieri: de la mythologie de l'histoire au volontarisme en tant qu'esthétique et anthropologie, pp. 323-333). J.M. VALENTIN (Lessing critique de Corneille: de 'Rodogune' à la théorie de la catharsis, pp. 339-351) е B. FRANCO (Corneille et les romantiques allemands: modèle classique et contre-modèle espagnol, pp. 353-360) affrontano la ricezione di Corneille in Germania, il primo analizzando la posizione di Lessing e il secondo quella dei romantici tedeschi.

7 All'interno della nona sezione, «De la tragédie à l'Opéra», E. MINEL evidenzia come l'opéra del xviII secolo riscopra l'idealismo pastorale del teatro corneliano (Opéras haendeliens et tragédies de Corneille: à propos de Pertharite/Rodelinda, Théodore/Theodora, La mort de Pompée/Julio Cesare. L'opéra comme repreneur de l'idéalisme pastoral subsistant au cœur de la tragédie cornélienne, pp. 363-386), mentre J.-P. CHAUVEAU mette diacronicamente in parallelo Cinna, il cui sottotitolo è La Clémence d'Auguste, e La Clemenza di Tito di Mozart (De Corneille à Mozart à travers Métastase: une rencontre imaginaire à cent cinquante ans de distance, ou de "Cinna" (1641) à "La Clémence de Titus" (1791), pp. 387-398). Dell'uso invece che i librettisti hanno fatto delle tragedie di Corneille si occupa B. DIDIER (Les tragédies de Pierre Corneille et l'Opéra (XVIII ${ }^{e}$-XIX ${ }^{e}$ siècles), pp. 403-410).

Della decima e ultima sezione, «Corneille en notre siècle», fanno parte i contributi di $\mathrm{H}$. SAWECKA (De Corneille à Pirandello ou le théâtre du point de vue de l'imaginaire, pp. 417-425) e di C. RIZZA (À propos d'une récente mise en scène de l'“Illusion comique", pp. 427-434), dai quali emerge con evidenza l'attualità del teatro corneliano. Entrambi dedicati all'Illusion comique, il primo vi rintraccia un «pirandellisme avant la lettre» (p. 418), mentre il secondo ha per argomento la recente messa in scena (stagione teatrale 2004/2005) da parte del Teatro Stabile di Genova della pièce corneliana nella traduzione italiana di Edoardo Sanguineti.

Chiude il volume J. MESNARD (Conclusion: Corneille l'Européen, pp. 439-451) che, riprendendo le fila dei diversi interventi che hanno animato il Convegno, conclude ritornando sul postulato di partenza, la portata cioè europea del drammaturgo e della sua produzione, ancora oggi in grado di affascinare il pubblico contemporaneo. 